\title{
ON A PROBLEM CONNECTED WITH BETA AND GAMMA DISTRIBUTIONS
}

BY

R. G. LAHA(1)

1. Introduction. The random variable $X$ is said to have a Gamma distribution $G(x ; \theta, \alpha)$ if

$$
P(X \leqq x)=G(x ; \theta, \alpha)= \begin{cases}\int_{0}^{x} \frac{\theta^{\alpha}}{\Gamma(\alpha)} u^{\alpha-1} e^{-\theta u} d u & \text { for } x>0, \\ 0 & \text { for } x \leqq 0,\end{cases}
$$

where $\theta>0, \alpha>0$. Let $X$ and $Y$ be two independently and identically distributed random variables each having a Gamma distribution of the form (1.1). Then it is well known [1, pp. 243-244], that the random variable $W=X /(X+Y)$ has a Beta distribution $B(w ; \alpha, \alpha)$ given by

$$
P(W \leqq w)=B(w ; \alpha, \alpha)= \begin{cases}0 & \text { for } w \leqq 0, \\ \int_{0}^{w} \frac{\Gamma(2 \alpha)}{\Gamma(\alpha) \Gamma(\alpha)} u^{\alpha-1}(1-u)^{\alpha-1} d u & \text { for } 0<w<1, \\ 1 & \text { for } w \geqq 1 .\end{cases}
$$

Now we can state the converse problem as follows:

Let $X$ and $Y$ be two independently and identically distributed random variables having a common distribution function $F(x)$. Suppose that $W=X /(X+Y)$ has a Beta distribution of the form (1.2). Then the question is whether $F(x)$ is necessarily a Gamma distribution of the form (1.1).

This problem was posed by Mauldon in [9]. He also showed that the converse problem is not true in general and constructed an example of a non-Gamma distribution with this property using the solution of an integral equation which was studied by Goodspeed in [2]. In the present paper we carry out a systematic investigation of this problem. In $\S 2$, we derive some general properties possessed by this class of distribution laws $F(x)$. In $\S 3$, we give some analytical lemmas. In $\$ 4$, we make use of the lemmas and deduce a characterization of a class of distribution laws having moments of a certain order. In the final section, we construct some further examples of distribution functions $F(x)$.

Received by the editors May 25, 1960 and, in revised form, June 7, 1963.

(1) This work was supported by the National Science Foundation through Grants NSF-G 4220 and NSF-G 9968. 
2. Some general properties of distribution laws. We first prove a lemma which is instrumental in the proof of the subsequent theorem.

LEMMA 2.1. Let $X$ and $Y$ be two independently and identically distributed random variables with a common distribution function $F(x)$ which is continuous at the point $x=0$. Suppose that the quotient $V=X / Y$ has a one-sided distribution $F_{0}(v)$ such that $F_{0}(v)=0$ for $v \leqq 0$. Then $X$ has also a one-sided distribution.

Proof. As usual we assume that each of the distribution functions $F(x)$ and $F_{0}(v)$ is everywhere continuous to the right so that for every $v>0$, we have

$$
\begin{aligned}
F_{0}(0)-F_{0}(-v)= & \operatorname{Prob}(-v<X / Y<0) \\
= & \int_{0}^{\infty}[F(0)-F(-v y)] d F(y) \\
& +\int_{-\infty}^{0}[F(-v y-0)-F(0)] d F(y) .
\end{aligned}
$$

From the conditions of the lemma we have $F_{0}(0)=F_{0}(-v)=0$ for every $v>0$ so that (2.1) gives

$$
\begin{array}{r}
\int_{0}^{\infty}[F(0)-F(-v y)] d F(y)=0, \\
\int_{-\infty}^{0}[F(-v y-0)-F(0)] d F(y)=0
\end{array}
$$

holding for all $v>0$. Then it follows immediately from (2.2a) that either $F(y)=0$ for all $y \leqq 0$ or $F(y)=1$ for all $y \geqq 0$. It is also easy to verify that the relation (2.2b) is satisfied in either of the above cases. This completes the proof of the lemma. We next prove the following theorem:

THEOREM 2.1. Let $X$ and $Y$ be two independently and identically distributed random variables with a common distribution function $F(x)$. Let the random variable $W=X /(X+Y)$ follow the Beta distribution of the form (1.2). Then $F(x)$ has the following general properties:

(1) Either $F(x)=0$ for $x \leqq 0$ or $F(x)=1$ for $x \geqq 0$,

(2) $F(x)$ is absolutely continuous and has a continuous probability density function $p(x)=F^{\prime}(x)>0$.

Proof. It is easy to verify from the conditions of the theorem that the quotient $V=X / Y=W /(1-W)$ has the distribution function $F_{0}(v)$ given by 


$$
F_{0}(v)= \begin{cases}\int_{0}^{v} \frac{\Gamma(2 \alpha)}{\Gamma(\alpha) \Gamma(\alpha)} \frac{u^{\alpha-1}}{(1+u)^{2 \alpha}} d u & \text { for } v>0 \\ 0 & \text { for } v \leqq 0\end{cases}
$$

Thus property (1) follows immediately from Lemma 2.1. Therefore, we can assume without any loss of generality that $F(x)=0$ for $x \leqq 0$, that is, the random variable $X$ is positive. After some elementary integration we can easily derive from (2.3) the characteristic function of the distribution of $\ln V$ as:

$$
E\left(e^{i t \ln V}\right)=\frac{\Gamma(\alpha+i t) \Gamma(\alpha-i t)}{\Gamma(\alpha) \Gamma(\alpha)} .
$$

We denote the characteristic function of the distribution of $\ln X$ by $\psi(t)$ and thus obtain the basic equation

$$
\psi(t) \psi(-t)=\frac{\Gamma(\alpha+i t) \Gamma(\alpha-i t)}{\Gamma(\alpha) \Gamma(\alpha)}
$$

holding for all real $t$. Then using the elementary property of the Gamma function we can at once verify that $\int_{-\infty}^{\infty}|\psi(t)| d t<\infty$, that is, the characteristic function $\psi(t)$ is absolutely integrable. Then using the Fourier-inversion theorem we conclude that the distribution function of $\ln X$ is absolutely continuous and has a continuous probability density function. Then it follows easily that $F(x)$ is also absolutely continuous and has a continuous probability density function $p(x)=F^{\prime}(x)>0$.

3. Some analytical lemmas. In this section we discuss some analytical lemmas which are instrumental in the proofs of the subsequent theorems.

LEMMA 3.1. Let $f(z)$ be an analytic characteristic function which is regular in a certain horizontal strip of maximum width containing the real axis. Then the purely imaginary points on the boundary of this strip of regularity are singular points of $f(z)$.

This lemma is due to Lévy [5]. A proof of this lemma is given by Lukacs in [8].

LEMMA 3.2. Let $f(z)$ be a decomposable characteristic function which is regular in a strip $-\beta<\operatorname{Im} z<\beta(\beta>0)$ of the complex $z$-plane. Let $f_{1}(z)$ be a factor of $f(z)$. Then the characteristic function $f_{1}(z)$ is also regular at least in the same strip.

This lemma on the factorization of analytic characteristic functions is due to Raikov [10].

LEMMA 3.3. Let $R(z)$ be an entire function of order unity having only purely imaginary zeros and $R(0)=1$. Then its reciprocal $1 / R(z)$ is always a characteristic function. 
The proof of this lemma is given in $[6$, p. 140].

LEMMA 3.4. Let $f(z)$ be an analytic characteristic function and suppose that $f_{0}(z)=[f(z)+f(-z)] / 2$ and $f_{1}(z)=[f(z)-f(-z)] / 2$ are respectively the even and odd parts off $f(z)$. Let $\rho, \rho_{0}$ and $\rho_{1}$ denote respectively the radii of convergence of Maclaurin series for $f(z), f_{0}(z)$ and $f_{1}(z)$. Then $\rho=\rho_{0}=\rho_{1}$.

This lemma has been proved by the author in [3].

LEMMA 3.5. Let $f(z)$ be the characteristic function of an infinitely divisible distribution which is regular in a certain horizontal strip. Then $f(z)$ has no zeros inside its strip of regularity and therefore $\phi(z)=\ln f(z)$ is defined and regular in the same strip. Moreover, the function $\theta(z)=\phi^{\prime \prime}(z) / \phi^{\prime \prime}(0)$ is also a characteristic function which is regular in the same strip.

The proof of this lemma is given in [8].

LEMMA 3.6. Let $p(x)$ be a continuous non-negative function of the real variable $x$. Let the integral $\int_{0}^{\infty} x^{v} p(x) d x$ ( $v$ real) exist for all $v$ in the interval $0<v<V(V>0)$. Then the integral $I(z)=\int_{0}^{\infty} x^{-i z} p(x) d x$ as a function of the complex variable $z$ is regular in the strip $0<\operatorname{Im} z<V$ of the upper half-plane.

This lemma has been proved by the author in [4]. As a special case of this lemma, we note that if the integral $\int_{0}^{\infty} x^{v} p(x) d x$ exists for all real $v>0$, then the integral $I(z)$ is regular throughout the upper half-plane $\operatorname{Im} z>0$.

LEMMA 3.7. Let the distribution function $F(x)$ of a positive random variable $X$ be absolutely continuous and have a continuous probability density function $p(x)=F^{\prime}(x)>0$. Let $F(x)$ possess finite absolute moments up to a certain order $\delta$ $(\delta>0$ not necessarily an integer $)$. Let $\psi(t)=E\left(e^{i t \ln x}\right)$ denote the characteristic function of the distribution of $\ln X$. Then the function

$$
\psi(-z)=E\left(e^{-i z \ln X}\right)
$$

considered as a function of the complex variable $z$ is regular in the strip $0<\operatorname{Im} z<\delta+\varepsilon(\varepsilon>0$ may be a suficiently small number $)$.

Conversely if the function $\psi(-z)$ is regular in the strip $0<\operatorname{Im} z<\delta+\varepsilon$ $(\varepsilon>0$ arbitrarily small), then $F(x)$ has finite absolute moments up to order $\delta$.

Proof. Since the distribution function $F(x)$ has finite absolute moments up to order $\delta$, the integral $\int_{0}^{\infty} x^{v} p(x) d x$ exists for all real $v$ in the interval $0<v<\delta+\varepsilon$ (where $\varepsilon$ may be an arbitrarily small positive number). We further note that

$$
\psi(-z)=E\left(e^{-i z \ln x}\right)=\int_{0}^{\infty} x^{-i z} p(x) d x .
$$

Then the proof follows at once from Lemma 3.6. The proof of the converse statement is immediate. 
As a special case of Lemma 3.7, it follows that if $F(x)$ has finite moments of all orders, then the function $\psi(-z)$ is regular throughout the upper half-plane $\operatorname{Im} z>0$.

4. A characterization of a class of distribution laws. In this section, we derive a characterization of a class of distribution laws having finite moments up to a certain order. Then we shall obtain a characterization of the Gamma distribution as a particular case. First, we prove the following theorem:

THEOREM 4.1. Under the same conditions as in Theorem 2.1, the distribution function $F(x)$ of the random variable $X$ always has finite absolute moments of order $\gamma$ where $0<\gamma<\alpha$.

Proof. As in Theorem 2.1, let $\psi(t)=E\left(e^{i t \ln x}\right)$ denote the characteristic function of the distribution of $\ln X$. Then $\psi(t)$ satisfies the basic equation (2.4) for real values of $t$. For further investigation we have to study the analytical behaviour of the function $\Gamma(\alpha+i z)$ in the complex $z$-plane. It is known that the function $1 / \Gamma(\alpha+i z)$ is an entire function of order unity which is real on the imaginary axis and has simple zeros at the points:

$$
z=i \gamma_{r} \text { where } \gamma_{r}=\alpha+r, \quad r=0,1,2, \cdots,
$$

all located on the imaginary axis in the upper half-plane. Therefore, we can use the factorization theorem of Hadamard [11, p. 250] and obtain

$$
\frac{\Gamma(\alpha)}{\Gamma(\alpha+i z)}=e^{i \rho z} \prod_{r=0}^{\infty}\left(1-\frac{z}{i \gamma_{r}}\right) e^{z / i \gamma_{r}} .
$$

Here it is easy to verify that $\rho \neq 0$ is a real constant. Similarly the function $1 / \Gamma(\alpha-i z)$ is an entire function of order unity having only purely imaginary zeros at the points:

$$
z=-i \gamma_{,} \text {where } \gamma_{r}=\alpha+r, \quad r=0,1,2, \cdots .
$$

Therefore it has a canonical representation similar to (4.1). Thus we have for complex values of $z$

$$
\frac{\Gamma(\alpha+i z) \Gamma(\alpha-i z)}{\Gamma(\alpha) \Gamma(\alpha)}=\prod_{r=0}^{\infty} \frac{1}{\left(1+z^{2} / \gamma_{r}^{2}\right)}
$$

where $\gamma_{r}=\alpha+r(\alpha>0, r=0,1,2, \cdots)$.

Then we conclude at once from the relation (4.2) that the characteristic function on the right-hand side of (2.4) can be continued in the complex $z$-plane and this function as a function of the complex variable $z$ is regular in the strip $|\operatorname{Im} z|<\alpha$. Then we applyLemma 3.2 on the factorization of analytic characteristic functions to the relation (2.4) and deduce at once that the function $\psi(t)$ can also be con- 
tinued in the complex $z$-plane and the function $\psi(z)$ is regular at least in the strip $|\operatorname{Im} z|<\alpha$. Thus $\psi(-z)$ is also regular in the strip $|\operatorname{Im} z|<\alpha$ and the proof follows at once from Lemma 3.7.

THEOREM 4.2. Under the same conditions as in Theorem 2.1, let the distribution function $F(x)$ have finite absolute moments up to order $\alpha+\delta(\delta \geqq 0)$. Let $\psi(t)$ denote the characteristic function of the distribution of $\ln X$, then the function $\psi(-z)$, as a function of the complex variable $z$, is regular in the strip $-\alpha<\operatorname{Im} z<\alpha+\delta+\varepsilon(\varepsilon>0$ sufficiently small). Conversely, if the function $\psi(-z)$ is regular in the strip $-\alpha<\operatorname{Im} z<\alpha+\delta+\varepsilon$, then the distribution function $F(x)$ has finite absolute moments up to order $\alpha+\delta$.

The proof of this theorem follows immediately when we combine the results of Lemma 3.7 and Theorem 4.1. As a special case of this theorem, we note that if the distribution function $F(x)$ has finite moments of all orders, then the function $\psi(-z)$ is regular throughout the region $\operatorname{Im} z>-\alpha(\alpha>0)$ of the complex $z$-plane.

First we discuss a method of constructing a class of infinitely many distribution functions $F(x)$ having finite absolute moments up to a certain order $\alpha+\delta(\delta \geqq 0)$, where the random variable $W=X /(X+Y)$ has a Beta distribution of the form (1.2) For this purpose we examine more closely the basic equation

$$
\psi(z) \psi(-z)=\prod_{r=0}^{\infty} \frac{1}{\left(1+z^{2} / \gamma_{r}^{2}\right)}
$$

where $\gamma_{r}=\alpha+r(\alpha>0 ; r=0,1,2, \cdots)$, assuming that (4.3) holds for all complex values of $z$ in the strip $|\operatorname{Im} z|<\alpha$. It is also easy to verify that the condition $\sum_{r=0}^{\infty} 1 / \gamma_{r}^{2}<\infty$ is satisfied. We divide the entire set of non-negative integers $(0,1,2, \cdots)$ into two mutually exclusive and exhaustive subsets and denote by

$$
(r)=\left(r_{1}, r_{2}, r_{3}, \cdots\right)
$$

an arbitrary subset of non-negative integers, finite or denumerable, and by

$$
(s)=\left(s_{1}, s_{2}, s_{3}, \cdots\right)
$$

the corresponding complementary subset such that

$$
(r)+(s)=(0,1,2, \cdots) \text {. }
$$

As a special case, any one of these subsets $(r)$ and $(s)$ may constitute the whole set of non-negative integers $(0,1,2, \cdots)$. In such a case the other subset reduces to an empty set. With the sets $(r)$ and $(s)$ so defined, we construct the entire functions of order unity as follows: 


$$
\left\{\begin{array}{l}
P(z)=\prod_{j=1}^{\infty}\left(1-\frac{z}{i \gamma_{r_{j}}}\right) e^{z / i \gamma_{r_{j}}}, \\
Q(z)=\prod_{j=1}^{\infty}\left(1-\frac{z}{i \gamma_{s_{j}}}\right) e^{z / i \gamma_{s_{j}}}
\end{array}\right.
$$

where $\gamma_{r_{j}}=\alpha+r_{j}$ and $\gamma_{s_{j}}=\alpha+s_{j}$.

It is then easy to verify that

$$
P(z) Q(z) P(-z) Q(-z)=\prod_{r=0}^{\infty}\left(1+\frac{z^{2}}{\gamma_{r}^{2}}\right) .
$$

We now write

$$
\psi(z)=\frac{e^{i \beta z}}{P(z) Q(-z)}
$$

where $P(z)$ and $Q(z)$ are already defined in (4.4) and $\beta$ is an arbitrary real number. We verify immediately from Lemma 3.3 that $\psi(z)$ is a characteristic function. We also note that in view of the relation (4.5) the characteristic function $\psi(z)$ which is regular in a certain horizontal strip containing the real axis satisfies the basic equation (4.3). Thus the function $\psi(z)$ yields a distribution function $F(x)$ with the desired property.

We next discuss the arrangement of elements in the sets $(r)$ and $(s)$ when it is given that the distribution function $F(x)$ has finite absolute moments up to order $\alpha+\delta(\delta \geqq 0)$. Without any loss of generality, we can assume that the integer elements in the sets $(r)$ and $(s)$ are arranged in increasing order of magnitude. It is easy to verify after some elementary arguments that in this case the arrangement of the elements in the sets $(r)$ and $(s)$ is as follows:

$$
\begin{aligned}
& (r)=(0,1,2, \cdots[\delta] \cdots), \\
& (s)=([\delta]+1, \cdots),
\end{aligned}
$$

that is, the first $[\delta]+1$ elements of the set $(r)$ are $0,1,2, \cdots[\delta]$ and the first element of the set $(s)$ is $[\delta]+1$, while the arrangement of the remaining integers in the sets $(r)$ and $(s)$ is left entirely arbitrary. Here $[\delta]$ denotes the largest integer contained in $\delta$. We can now summarize the main result in the form of the following theorem:

THEOREM 4.3. Let $X$ be a positive random variable such that the characteristic function $\psi(z)$ of the distribution of $\ln X$ is given by (4.6) where $P(z)$ and $Q(z)$ are defined as in (4.4). Let the integer elements in both sets $(r)$ and $(s)$ be arranged in increasing order of magnitude and further satisfy. the condition in (4.7). Any such construction of $\psi(z)$ yields a distribution function $F(x)$ having finite absolute moments up to order $\alpha+\delta(\delta \geqq 0)$ where the random variable $X /(X+Y)$ has a Beta distribution of the form (1.2). 
From now on we denote this class of distribution functions by $C_{\alpha+\delta}$. We are now in a position to prove the following theorem on a characterization of the class $C_{\alpha+\delta}$.

THEOREM 4.4. Let the following two conditions be satisfied in addition to the restrictions of Theorem 2.1:

(1) The distribution function $F(x)$ of the random variable $X$ has finite absolute moments up to order $\alpha+\delta(\delta \geqq 0)$.

(2) The random variable $\ln X$ has an infinitely divisible distribution.

Then $F(x)$ belongs to the class $C_{\alpha+\delta}$.

Proof. We write $\psi_{0}(z)=1 / P(z) Q(-z)$ where $P(z)$ and $Q(z)$ are defined in (4.4). First we note that the characteristic function $\psi_{0}(z)$ is infinitely divisible, as it is obtained as the limit of a sequence of infinitely divisible characteristic functions. Let the integer elements in the sets $(r)$ and $(s)$ corresponding to $\psi_{0}(z)$ be arranged in increasing order of magnitude and further satisfy the condition (4.7). Then $\psi_{0}(-z)$ is regular in the strip $-\alpha<\operatorname{Im} z<\alpha+[\delta]+1$ and has no zeros inside this strip. It is also easy to verify from the conditions of the theorem that the characteristic function $\psi(-z)=E\left(e^{-i z \ln x}\right)$ is also regular in the strip $-\alpha<\operatorname{Im} z<\alpha+[\delta]+1$ and has no zeros inside this strip. We write

$$
\psi(z)=\psi_{0}(z) \theta(z)
$$

and verify easily that the function $\theta(-z)$ is also regular in the strip $-\alpha<\operatorname{Im} z<\alpha+[\delta]+1$ and has no zeros inside this strip. We also note that each of the functions $\psi(z)$ and $\psi_{0}(z)$ satisfies the basic equation (4.3) so that we obtain

$$
\theta(z) \theta(-z)=1
$$

From the relation (4.9) we can verify easily that the function $\ln \theta(z)$ is also regular in the strip $|\operatorname{Im} z|<\alpha+[\delta]+1$. We further conclude from (4.9) that $\ln \theta(z)$ is an odd function, that is,

$$
\ln \theta(-z)=-\ln \theta(z)
$$

Next we multiply both sides of $(4.8)$ by $\psi_{0}(-z)$ and obtain

$$
\phi(z)=\phi_{0}(z) \theta(z)
$$

where $\phi(z)=\psi(z) \psi_{0}(-z)$ and $\phi_{0}(z)=\psi_{0}(z) \psi_{0}(-z)$.

Here we note that each of the functions $\phi(z)$ and $\phi_{0}(z)$ is an infinitely divisible characteristic function and is regular in the strip $|\operatorname{Im} z|<\alpha$. We further remark that the function $\phi_{0}(z)$ is an even characteristic function. We now set 


$$
\begin{aligned}
& f(z)=\frac{1}{c} \cdot \frac{d^{2}}{d z^{2}} \ln \phi(z) \\
& f_{0}(z)=\frac{1}{c} \cdot \frac{d^{2}}{d z^{2}} \ln \phi_{0}(z) \\
& f_{1}(z)=\frac{1}{c} \cdot \frac{d^{2}}{d z^{2}} \ln \theta(z)
\end{aligned}
$$

where

$$
c=\left[\frac{d^{2}}{d z^{2}} \ln \phi(z)\right]_{z=0}
$$

and deduce easily from the equation (4.11) the relation

$$
f(z)=f_{0}(z)+f_{1}(z) .
$$

We now use Lemma 3.5 and verify easily from (4.12) that the functions $f_{0}(z)$ and $f_{1}(z)$ are respectively the even and odd parts of the characteristic function $f(z)$. Here $f_{0}(z)$ is only regular in the strip $|\operatorname{Im} z|<\alpha$, while $f_{1}(z)$ is regular in the larger strip $|\operatorname{Im} z|<\alpha+[\delta]+1$. But according to Lemma 3.4, this is not possible. Therefore $f_{1}(z) \equiv 0$ for all $z$ so that $\ln \theta(z)=i \beta z$. We further note that each of the functions $\phi(z)$ and $\phi_{0}(z)$ are real on the imaginary axis. Hence it follows at once from (4.11) that $\theta(z)$ must also be real for purely imaginary values of $z$ so that

$$
\theta(z)=e^{i \beta z}
$$

where $\beta$ is an arbitrary real number. Thus we obtain finally from (4.8) the equation

$$
\psi(z)=\frac{e^{i \beta z}}{P(z) Q(-z)}
$$

where $P(z)$ and $Q(z)$ are defined in (4.4). Then the proof of the theorem follows immediately, since the integer elements in the sets $(r)$ and $(s)$ corresponding to $P(z)$ and $Q(z)$ in (4.13) satisfy the condition in (4.7). The following characterization of the Gamma distribution is an immediate consequence of Theorem 4.4.

THEOREM 4.5. Let the following conditions be satisfied in addition to the restrictions of Theorem 2.1:

(1) The distribution function $F(x)$ of the random variable $X$ has finite absolute moments of all orders.

(2) The random variable $\ln X$ has an infinitely divisible distribution. Then $F(x)$ is a Gamma distribution of the form (1.1).

Proof. It follows at once from Theorem 4.4 that under the conditions of the theorem the set $(r)$ constitutes the whole set of non-negative integers $(0,1,2, \cdots)$ while the set $(s)$ reduces to an empty set. As a consequence we obtain from (4.1) the relation 


$$
\psi(z)=\frac{\Gamma(\alpha+i z)}{\Gamma(\alpha)} e^{i \gamma z}
$$

holding for all complex $z$ in the region $\operatorname{Im} z<\alpha$, where $\gamma$ is an arbitrary real number. We next substitute $z=-i v(v>0$ real $)$ in (4.14) and obtain

$$
\psi(-i v)=\int_{0}^{\infty} x^{v} p(x) d x=\frac{\Gamma(\alpha+v)}{\Gamma(\alpha)} e^{\gamma v} .
$$

Finally we set $v=k(k>0$ an integer $)$ and obtain

$$
\mu_{k}=\int_{0}^{\infty} x^{k} p(x) d x=\frac{\Gamma(\alpha+k)}{\Gamma(\alpha)} \frac{1}{\theta^{k}} \quad\left(\theta=e^{-\gamma}>0\right) .
$$

Here $\mu_{k}$ is the moment of order $k$ of the distribution function $F(x)$. The proof of Theorem 4.5 follows immediately from the fact that the moments in (4.16) uniquely determine the Gamma distribution $G(x ; \theta, \alpha)$ given in (1.1).

5. Further examples. In this section, we shall construct another class of infinitely many characteristic functions $\psi(z)$ which satisfy the basic equation (4.3) and for which the corresponding distribution functions $F(x)$ have finite absolute moments up to order $\alpha+\delta(\delta \geqq 0)$. But we shall show that the characteristic functions $\psi(z)$ so constructed are not infinitely divisible and consequently we shall verify that the condition (2) of Theorem 4.4 as well as of Theorem 4.5 is essential.

For this purpose we require the following lemma:

LEMMA 5.1. Let

$$
f(t)=\frac{\left(1+\frac{i t}{\mu}\right)\left(1+\frac{i t}{\bar{\mu}}\right)}{\left(1-\frac{i t}{\gamma}\right)\left(1-\frac{i t}{\mu}\right)\left(1-\frac{i t}{\bar{\mu}}\right)}
$$

where $\mu=\gamma+i \delta, \bar{\mu}=\gamma-i \delta$ and $\gamma>0 ; \delta>0$ are both real. Then $f(t)$ is always a characteristic function whenever the relation $\delta \geqq 2 \sqrt{ } 2 \gamma$ is satisfied.

The proof follows from a more general result on rational characteristic functions given by Lukacs in [7].

We next define the quantities:

$$
\begin{cases}\gamma_{r_{j}}=\alpha+r_{j} ; & j=1,2, \cdots, N_{1} ; N_{1}+1, \cdots, \\ \delta_{r_{j}} \geqq 2 \sqrt{ } 2 \gamma_{r_{j}} ; & j=1,2, \cdots, N_{1}, \\ \mu_{r_{j}}=\gamma_{r_{j}}+i \delta_{r_{j}} ; & j=1,2, \cdots, N_{1}, \\ \bar{\mu}_{r_{j}}=\gamma_{r_{j}}-i \delta_{r_{j}} ; & j=1,2, \cdots, N_{1},\end{cases}
$$


and

$$
\begin{cases}\gamma_{s_{j}}=\alpha+s_{j} ; & j=1,2, \cdots, N_{2} ; N_{2}+1, \cdots, \\ \delta_{s_{j}} \geqq 2 \sqrt{ } 2 \gamma_{s_{j}} ; & j=1,2, \cdots, N_{2}, \\ \mu_{s_{j}}=\gamma_{s_{j}}+i \delta_{s_{j}} ; & j=1,2, \cdots, N_{2}, \\ \bar{\mu}_{s_{j}}=\gamma_{s_{j}}-i \delta_{s_{j}} ; & j=1,2, \cdots, N_{2},\end{cases}
$$

corresponding to the elements of the sets $(r)$ and $(s)$ where the positive integers $N_{1}$ and $N_{2}$ may be either finite or infinite. We verify easily that the condition $\sum_{r=0}^{\infty} 1 /\left|\mu_{r}\right|^{2}<\sum_{r=0}^{\infty} 1 / \gamma_{r}^{2}<\infty$ is satisfied.

We now construct the function $\psi(z)$ as

$$
\psi(z)=\prod_{j=1}^{N_{1}} \frac{\left(1+\frac{z}{i \mu_{r_{j}}}\right)\left(1+\frac{z}{i \bar{\mu}_{r_{j}}}\right) \bar{e}^{z / i \gamma_{r_{j}}}}{\left(1-\frac{z}{i \gamma_{r_{j}}}\right)\left(1-\frac{z}{i \mu_{r_{j}}}\right)\left(1-\frac{z}{i \bar{\mu}_{r_{j}}}\right)} \prod_{J=N_{1}+1}^{\infty} \frac{e^{-z / i \gamma_{r_{j}}}}{\left(1-\frac{z}{i \gamma_{r_{j}}}\right)}
$$

$$
\times \prod_{j=1}^{N_{2}} \frac{\left(1-\frac{z}{i \mu_{s_{j}}}\right)\left(1-\frac{z}{i \bar{\mu}_{s_{j}}}\right) \epsilon^{z / i \gamma_{s_{j}}}}{\left(1+\frac{z}{i \gamma_{s_{j}}}\right)\left(1+\frac{z}{i \mu_{s_{j}}}\right)\left(1+\frac{z}{i \bar{\mu}_{s_{j}}}\right)} \prod_{j=N_{2}+1}^{\infty} \frac{e^{z / i \gamma_{s_{j}}}}{\left(1+\frac{z}{i \gamma_{s_{j}}}\right)} .
$$

If both the numbers $N_{1}$ and $N_{2}$ are finite we conclude immediately from Lemmas 3.3 and 5.1 that the function $\psi(z)$ in (5.3) is a characteristic function. On the other hand, if either of the numbers $N_{1}$ or $N_{2}$ is infinite, we can also verify easily that $\psi(z)$ is a characteristic function, using the continuity theorem of Lévy. We further note that the characteristic function $\psi(z)$ in (5.3) satisfies the basic equation (4.3) and the function $\psi(-z)$ is regular in the strip $-\gamma_{r_{1}}<\operatorname{Im} z<\gamma_{s_{1}}$. Thus in this case we can again obtain a theorem which is analogous to Theorem 4.3.Therefore we have constructed another class of infinitely many distribution functions $F(x)$ having finite moments up to a certain order and possessing the desired property. But we note at the same time that the characteristic function $\psi(z)$ constructed in (5.3) always has simple zeros at the points $z=-i \gamma_{r_{1}} \pm \delta_{r_{1}}$, that is, at the points $z=-i \alpha \pm \delta_{0}$ inside its strip of regularity. Consequently $\psi(z)$ cannot be the characteristic function of an infinitely divisible distribution. Proceeding exactly in the same way, one can construct an example of a distribution function which is not a Gamma distribution but has finite absolute moments of all orders where the random variable $X /(X+Y)$ has a Beta distribution of the form (1.2). But in such a case the random variable $\ln X$ does not have an infinitely divisible distribution so that the condition (2) of Theorem 4.5 is not satisfied. 


\section{REFERENCES} 1946.

1. H. Cramér, Mathematical methods of statistics, Princeton Univ. Press, Princeton, N.J.

2. F. M. C. Goodspeed, The relation between functions satisfying a certain integral equation and general Watson transforms, Canad. J. Math. 2 (1950), 223-237.

3. R. G. Laha, Une remarque sur les fonctions caractéristiques analytiques, C. R. Acad. Sci. Paris 249 (1959), 358-359.

4. - On a class of distribution functions where the quotient follows the Cauchy law, Trans. Amer. Math. Soc. 93 (1959), 205-215.

5. P. Lévy, L'arithmétique des lois de probabilité, J. Math. Pures Appl. 17 (1938), 17-39.

6. E. Lukacs and O. Szász, Certain Fourier transforms of distributions, Canad. J. Math. 3 (1951), 140-144.

7. E. Lukacs, Remarks concerning characteristic functions, Ann. Math. Statist. 28 (1957), 717-723.

8. —— Les fonctions caractéristiques analytiques, Ann. Inst. H. Poincaré 15 (1957), 217-251.

9. J. G. Mauldon, Characterizing properties of statistical distributions, Quart. J. Math. Oxford Ser. (2) 7 (1956), 155-160.

10. D. A. Raikov, On the decomposition of Gauss and Poisson laws, Izv. Akad. Nauk SSSR Ser. Mat. 2 (1938), 91-124. (Russian)

11. E. C. Titchmarsh, The theory of functions, Oxford Univ. Press, Oxford, 1939.

The Catholic University of America,

WASHINGTON, D.C. 